Subscriber access provided by Caltech Library

\title{
Communication
}

\section{Inorganic Phototropism in Electrodeposition of Se-Te}

\author{
Madeline C. Meier, Wen-Hui Cheng, Harry A Atwater, Nathan S. Lewis, and Azhar I. Carim
}

J. Am. Chem. Soc., Just Accepted Manuscript • DOI: 10.1021/jacs.9b10579 • Publication Date (Web): 07 Nov 2019

Downloaded from pubs.acs.org on November 7, 2019

\section{Just Accepted}

"Just Accepted" manuscripts have been peer-reviewed and accepted for publication. They are posted online prior to technical editing, formatting for publication and author proofing. The American Chemical Society provides "Just Accepted" as a service to the research community to expedite the dissemination of scientific material as soon as possible after acceptance. "Just Accepted" manuscripts appear in full in PDF format accompanied by an HTML abstract. "Just Accepted" manuscripts have been fully peer reviewed, but should not be considered the official version of record. They are citable by the Digital Object Identifier (DOI®). "Just Accepted" is an optional service offered to authors. Therefore, the "Just Accepted" Web site may not include all articles that will be published in the journal. After a manuscript is technically edited and formatted, it will be removed from the "Just Accepted" Web site and published as an ASAP article. Note that technical editing may introduce minor changes to the manuscript text and/or graphics which could affect content, and all legal disclaimers and ethical guidelines that apply to the journal pertain. ACS cannot be held responsible for errors or consequences arising from the use of information contained in these "Just Accepted" manuscripts. 
Many photosynthetic species, including plants such as palms, cabbage, and grass, as well as other organisms such as coral, exhibit phototropic growth, in which the growth of a biological system proceeds preferentially towards the time-averaged position of the sun..$^{1-4} \mathrm{~A}$ responsive phototropic growth process for inorganic materials that mimics natural phototropism may enable bottom-up, directed, and programmable generation of three-dimensional nanostructures and mesostructures in free space. Maskless photoelectrochemical growth of semiconducting Se-Te alloy films spontaneously produces ordered nanoscale features over macroscopic length scales, resulting in a variety of mesoscale film morphologies that are a function of the photoelectrochemical growth response of the discrete nanoscale features as well as the emergent behavior of the growing film. ${ }^{5-7}$ Electrochemical growth of Se-Te from $0.0200 \mathrm{M} \mathrm{SeO}_{2}, 0.0100 \mathrm{M} \mathrm{TeO}_{2}$, and $2.00 \mathrm{M}$ $\mathrm{H}_{2} \mathrm{SO}_{4}$ is accelerated by, and responsive to the wavelength, intensity, and polarization characteristics of, illumination with energy above the Se-Te band gap. For example, use of polarized light to stimulate film growth produces lamellae aligned along the optical polarization direction. Such growth phenomena are observed despite the use of an optically isotropic solution and substrate and an unstructured optical input, and the lack of any chemical or physical templating agents as well as any epitaxial relationship between the growing deposit and substrate. In

this work, we demonstrate the spatially directed, inorganic phototropic growth of isolated Se-Te deposits in response to manipulation of the direction of a spatially conformal, incoherent, and unpolarized beam of light.

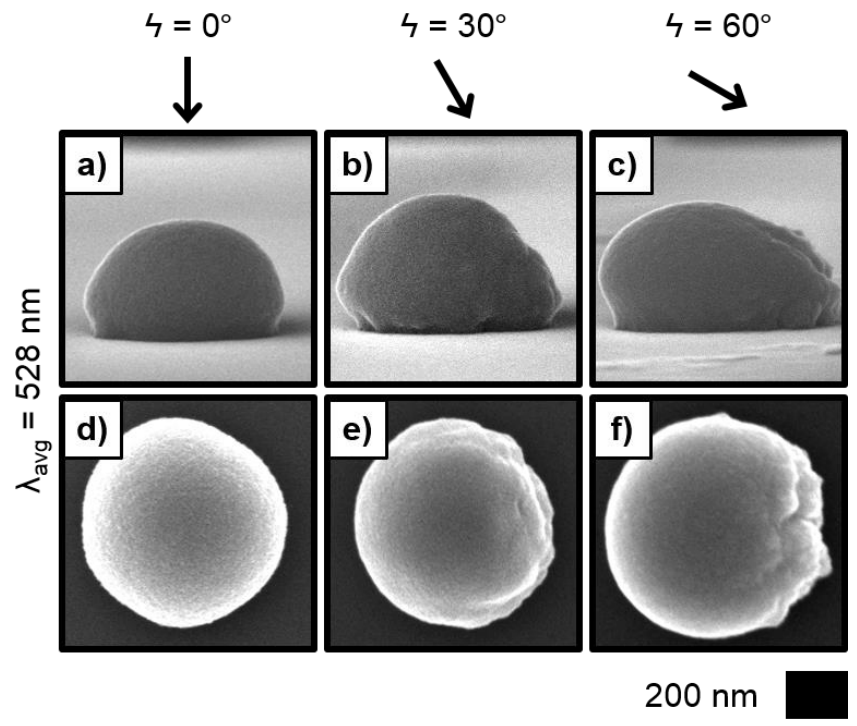

Figure 1. (a)-(c) Cross-sectional and (d)-(f) top-down SEMs representative of photoelectrodeposits generated with $\lambda_{\text {avg }}=$ $528 \mathrm{~nm}$ illumination incident at the indicated angle 4 from the substrate normal.

Se-Te was electrochemically grown under illumination on circular $\mathrm{Au}$ islands that were lithographically patterned onto an $\mathrm{n}^{+}-\mathrm{Si}$ substrate. The feature size and pitch of the $\mathrm{Au}$ islands were designed to minimize optical and electrochemical communication between adjacent regions of growth. Such isolation enabled investigation of the intrinsic material growth behavior independent of emergent phenomena, including array effects, which may dominate the growth characteristics of an unconstrained, extended film. ${ }^{7}$ Figure 1a-c presents representative crosssectional scanning-electron micrographs (SEMs) of isolated Se-Te deposits generated using a narrowband lightemitting diode (LED) source with an intensity-weighted average wavelength, $\lambda_{\text {avg, }}$ of $528 \mathrm{~nm}$, with the illumination incident at the indicated angle, 4 , from the surface 
normal. With $\zeta=0^{\circ}$ (normal incidence, Figure 1a), a symmetrical, hemispherical cross-section was observed. For $\zeta=30^{\circ}$ (Figure 1b) the deposit cross-section was mostly hemispherical, but exhibited some asymmetry in that greater mass was observed on the side of the incident illumination relative to the opposite side. For $\zeta=60^{\circ}$ (Figure 1c) a larger growth anisotropy was observed than for $\zeta=30^{\circ}$ (Figure 1b). Figure 1d-f presents top-down SEMs analogous to the cross-sections presented in Figure 1a-c. With $\zeta=0^{\circ}$ (Figure 1d) the deposit morphology was symmetrical, with an apparent circular projection. For both 4 $=30^{\circ}$ and $\zeta=60^{\circ}$ (Figure 1e and f) less mass addition, and increased apparent roughness, was observed on the far side of the deposits, away from the direction of incident illumination. Hence the growth of Se-Te deposits can be directed to mimic the natural phototropism observed in biological systems, wherein mass addition is directed asymmetrically to extend the structure along the path of the incident illumination.

In many plant systems that exhibit phototropic growth, including the model organism Arabidopsis, the magnitude of the natural phototropic response is a function of the illumination wavelength, with phototropic growth primarily observed in response to photosynthesis stimulated by short-wavelength visible light. ${ }^{2,8}$ The mechanism of natural phototropic growth has been explained by the molecular absorption necessary to drive the photochemical reactions that underpin the biological growth response, in conjunction with an auxin-related response that causes cells to elongate at locations farthest from the light. ${ }^{9-12}$ Herein, electrochemical growth using illumination

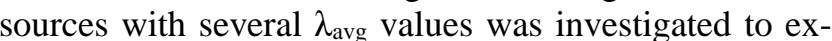
plore the wavelength sensitivity of Se-Te inorganic phototropic growth. Figure $2 \mathrm{a}-\mathrm{c}$ presents cross-sectional SEMs of deposits generated using a LED with $\lambda_{\text {avg }}=727$ $n m$ with varying values of $\zeta$. With $\zeta=0^{\circ}$ (Figure $2 \mathrm{a}$ ) a symmetrical, hemispherical cross-section was observed, similar to the case of normally incident $\lambda_{\text {avg }}=528 \mathrm{~nm}$ illumination (Figure 1a). For $\zeta=30^{\circ}$ (Figure 2b), some azimuthal asymmetry was observed in the deposits, with increased mass addition on the side of the incident illumination, but with less azimuthal asymmetry than growth using $\lambda_{\text {avg }}=528 \mathrm{~nm}$ at $\zeta=30^{\circ}$ (Figure 1b). The deposit generated with $\zeta=60^{\circ}$ (Figure 2c) exhibited the same type of asymmetry as for $\zeta=30^{\circ}$ (Figure $2 \mathrm{~b}$ ) but with substantially greater azimuthal asymmetry, similar to the case of $\lambda_{\text {avg }}=528 \mathrm{~nm}$ incident at $\zeta=60^{\circ}$ (Figure 1c). Figures $2 \mathrm{~d}-\mathrm{f}$ present data analogous to that presented in Figure $2 \mathrm{a}-\mathrm{c}$ but for deposits generated using a LED with $\lambda_{\text {avg }}$ $=843 \mathrm{~nm}$. In all three cases $\left(\zeta=0,30\right.$ and $\left.60^{\circ}\right)$ a symmetrical, hemispherical cross-section was observed. Similar to the behavior observed in natural systems, the magnitude of the inorganic phototropic response varied with wavelength, and asymmetry was not observable for growth stimulated by illumination having a sufficiently long wavelength (near-infrared).

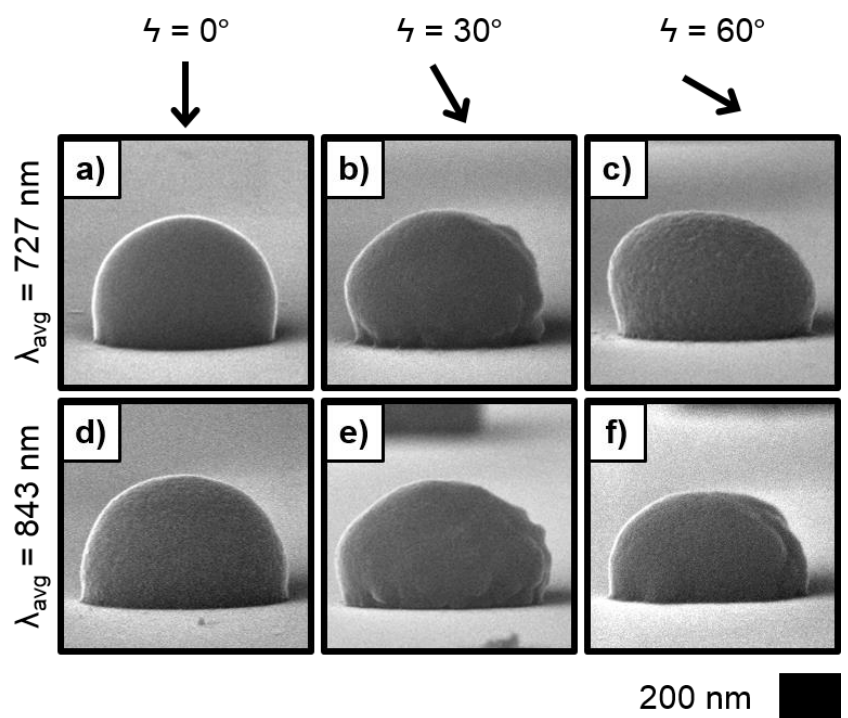

Figure 2. Cross-sectional SEMs representative of photoelectrodeposits generated with the indicated $\lambda_{\text {avg }}$ illumination incident at the indicated angle $\zeta$ from the substrate normal.

To gain insight into the relation between the material absorption and the observed phototropic response, light absorption simulations were performed using full-wave electromagnetic simulations. ${ }^{13}$ Model Se-Te structures about metallic islands in an electrolyte solution were used as representations of the morphologies that developed during photoelectrochemical growth. Analysis by energydispersive X-ray (EDX) spectroscopy and Raman spectroscopy (Table S1 and Figure S7) indicated that the composition of the photoelectrodeposited material was independent of the illumination inputs for the investigated parameter space. The materials properties used in the modeling were in accord with the composition of the material measured experimentally by EDX and Raman spectroscopies. The optical extinction coefficient of Se-Te alloys decreases with increasing wavelength from the visible regime to the near-infrared regime. ${ }^{14}$ Figure $3 \mathrm{a}-\mathrm{c}$ presents graphical representations of the light absorption profiles calculated for the model structure using $\lambda_{\text {avg }}=528 \mathrm{~nm}$ for $\zeta=0,30$ and $60^{\circ}$. For $\zeta=0^{\circ}$ (Figure $3 \mathrm{a}$ ) the absorption profile was symmetrical and most of the absorption was strongly localized near the growth (solution) interface. With $\zeta=30^{\circ}$ (Figure $3 \mathrm{~b}$ ) the absorption was also tightly confined near the solution interface but was asymmetrically distributed spatially, with greater absorption on the side of the incident illumination. A similar profile, but with a greater degree of angular asymmetry, was observed for $৬=60^{\circ}$ (Figure 3c). The extent of asymmetry in the absorption profiles correlated with the morphological anisotropies observed for deposits generated with the $\lambda_{\text {avg }}=$ $528 \mathrm{~nm}$ source (Figure 1). The greater surface roughness observed for deposits generated with the $\lambda_{\text {avg }}=528 \mathrm{~nm}$ source and $\zeta=30$ and $60^{\circ}$ on the far side, away from the direction of illumination incidence, relative to the illuminated side, is similar to the roughness observed for a deposit generated in the dark (Figure S5). This observation 
suggests that the absorption of light by the deposit is sufficient to attenuate light-directed growth on the far side and relatively there favor dark growth wherein mass addition may be dictated by electrochemical kinetics.

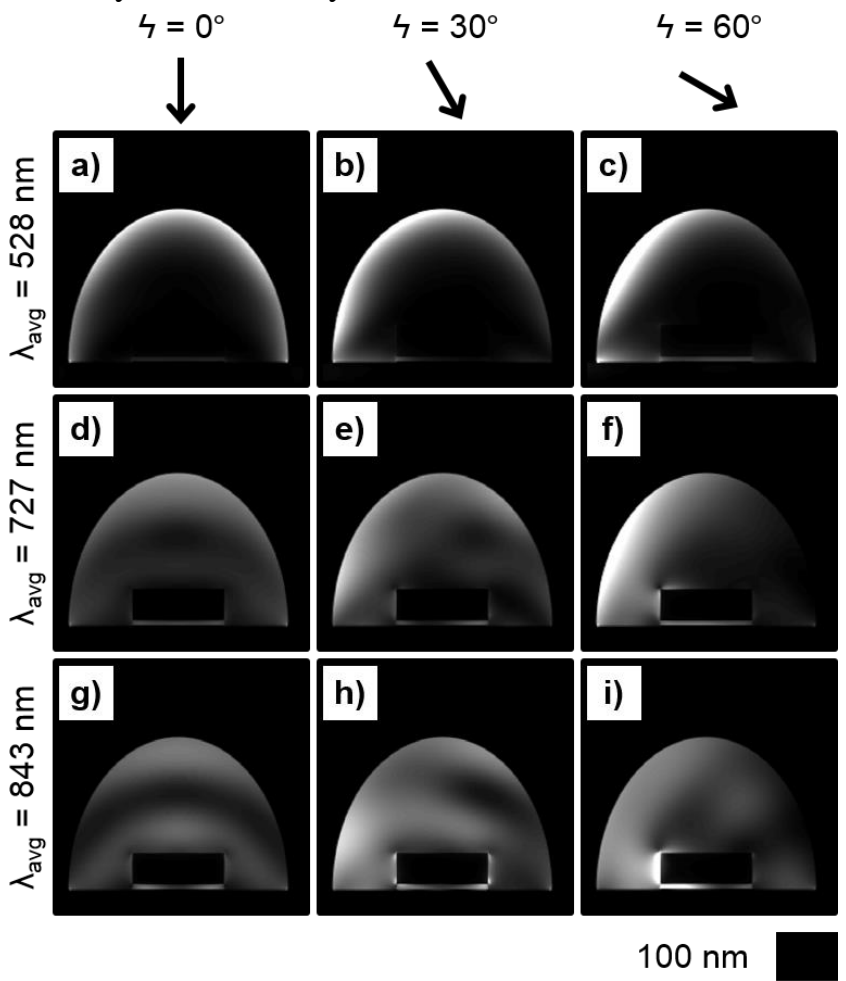

Figure 3. Simulated light absorption profiles in a model photoelectrodeposit morphology for illumination with the indicated $\lambda_{\text {avg }}$ incident at the indicated angle $\zeta$ from the substrate normal.

Figure 3d-f presents analogous data to Figure 3a-c but for calculations using $\lambda_{\text {avg }}=727 \mathrm{~nm}$. For $\zeta=0^{\circ}$ (Figure $3 \mathrm{~d})$ the profile was symmetric but exhibited less concentration of absorption along the solution interface than for $\lambda_{\text {avg }}=528 \mathrm{~nm}$ (Figure 3a). For $\zeta=30^{\circ}$ (Figure 3e) the profile showed some absorption concentration on side of the incident illumination but was more azimuthally isotropic than the profile for $\lambda_{\text {avg }}=528 \mathrm{~nm}$ with $\zeta=30^{\circ}$ (Figure $3 \mathrm{~b}$ ). With $\zeta=60^{\circ}$ (Figure 3f), the absorption was asymmetrically concentrated on the same side of the normal as the incident illumination but was not confined as strongly along the interface as for any angle of incidence with $\lambda_{\text {avg }}=528 \mathrm{~nm}$ (Figure 3a-c). The decreased optical concentration near the growth interface observed in the profiles for $\lambda_{\text {avg }}=727 \mathrm{~nm}$ relative to those for $\lambda_{\text {avg }}=528$ $\mathrm{nm}$ is consistent with the decrease in extinction coefficient of the Se-Te material as the wavelength increases. As for $\lambda_{\text {avg }}=528 \mathrm{~nm}$, the azimuthal symmetry observed in the absorption profiles for $\lambda_{\text {avg }}=727 \mathrm{~nm}$ (Figure $3 \mathrm{~d}-\mathrm{f}$ ) is consistent with the morphological anisotropy in the deposits generated with the same $\lambda_{\text {avg }}$ (Figure $2 \mathrm{a}-\mathrm{c}$ ).

Figure $3 g-i$ presents representations of absorption profiles similar to those presented in Figure $3 \mathrm{a}-\mathrm{c}$ and $\mathrm{d}-\mathrm{f}$ but for calculations using $\lambda_{\text {avg }}=843 \mathrm{~nm}$. For $\zeta=0^{\circ}$ (Figure
$3 \mathrm{~g})$ the profile is symmetric, but a similar magnitude of absorption is observed in both the apex of the structure near the solution interface and at the bottom of the structure near the substrate interface, in contrast to the profile observed for $\lambda_{\text {avg }}=528 \mathrm{~nm}$ with $\zeta=0^{\circ}$ (Figure $3 \mathrm{a}$ ). For $\zeta$ $=30^{\circ}$ and $60^{\circ}$ (Figure $3 \mathrm{~h}$ and i), some azimuthal asymmetry in the absorption profiles was observed, with the absorption biased toward the side of the incident illumination. However, the overall localization of the absorption was lower than observed in the analogous cases with $\lambda_{\text {avg }}=727 \mathrm{~nm}$ (Figure 3e and $\mathrm{f}$ ). The low degree of optical concentration observed in the profiles for $\lambda_{\text {avg }}=843 \mathrm{~nm}$ (Figure 3g-i) is consistent with the lower material optical absorption at this wavelength than at $\lambda_{\text {avg }}=528 \mathrm{~nm}$ or $\lambda_{\text {avg }}$ $=727 \mathrm{~nm}$, and is moreover consistent with the lack of morphological anisotropy observed experimentally for deposits generated with the $\lambda_{\mathrm{avg}}=843 \mathrm{~nm}$ source. The computational data, in conjunction with experimental results, thus indicates that the phototropic response arises from spatially anisotropic absorption in the growing deposits, which in turn promotes locally elevated rates of electrochemical growth where absorption is high, resulting in directional growth. ${ }^{6,13}$ A phototropic response was not observed when the optical absorption was predominantly spatially isotropic, such as for $\lambda_{\text {avg }}=843 \mathrm{~nm}$ (Figure $3 g-i)$. Due to low optical absorption and thus high optical penetration under these conditions, the isotropic absorption resulted in spatially isotropic rates of mass addition along the growth interface, effecting symmetric deposits.

The cumulative data presented herein demonstrates that inorganic phototropic growth, wherein material addition is directed towards an unstructured light beam in free space in analogy to the manner that many plants grow towards the sun, can be achieved via light-guided electrochemical growth of Se-Te. The directionality of the inorganic phototropic response is a function of direction of the illumination incidence whereas the magnitude of the response is dependent on both the incidence vector and the wavelength. Simulations of light absorption in model Se-Te deposits, combined with the experimental data, indicate that the phototropic response is a consequence of spatially anisotropic absorption in the evolving material. Techniques to capitalize on inorganic phototropic response to construct complex three-dimensional morphologies that may be difficult or impossible to generate via conventional lithographic means are now being investigated.

\section{ASSOCIATED CONTENT}

\section{Supporting Information.}

The Supporting Information is available free of charge on the ACS Publications website.

Details regarding experimental and modeling/simulation methods, additional scanning-electron mi- 
crographs, analysis of photoelectrodeposit elemental composition and structure, voltammetric data (PDF)

\section{AUTHOR INFORMATION}

\section{Corresponding Author}

*Email: nslewis@caltech.edu.

\section{ACKNOWLEDGMENT}

This work was supported by the National Science Foundation under Award Number DMR1905963. The authors gratefully acknowledge K. Hamann for insightful discussions and R. Gerhart, N. Hart, and B. Markowicz for assistance with photoelectrochemical cell fabrication. MCM acknowledges a Graduate Research Fellowship from the National Science Foundation.

\section{REFERENCES}

(1) Tomlinson, P. B. The Structural Biology of Palms; Oxford University Press: New York, 1990.

(2) Christie, J. M.; Murphy, A. S. Shoot phototropism in higher plants: New light through old concepts. Am. J. Bot. 2013, 100, 35-46.

(3) Iwase, A.; Sakai, K.; Suzuki, A.; van Woesik, R. Phototropic adjustment of the foliaceous coral Echinopora lamellosa in Palau. Estuar. Coast. Shelf Sci. 2008, 77, 672-678.

(4) Kaniewska, P.; Campbell, P. R.; Fine, M.; Hoegh-Guldberg, O. Phototropic growth in a reef flat acroporid branching coral species. $J$. Exp. Biol. 2009, 212, 662-667.
(5) Carim, A. I.; Batara, N. A.; Premkumar, A.; Atwater, H. A. Lewis, N. S. Self-Optimizing Photoelectrochemical Growth of Nanopatterned Se-Te Films in Response to the Spectral Distribution of Incident Illumination. Nano Lett. 2015, 15, 7071-7076.

(6) Carim, A. I.; Batara, N. A.; Premkumar, A.; Atwater, H. A.; Lewis, N. S. Polarization Control of Morphological Pattern Orientation During Light-Mediated Synthesis of Nanostructured Se-Te Films. ACS Nano 2016, 10, 102-111.

(7) Sadtler, B.; Burgos, S. P.; Batara, N. A.; Beardslee, J. A.; Atwater, H. A.; Lewis, N. S. Phototropic growth control of nanoscale pattern formation in photoelectrodeposited Se-Te films. Proc. Natl. Acad. Sci. U. S. A. 2013, 110, 19707-19712.

(8) Fankhauser, C.; Chory, J. Light Control of Plant Development. Annu. Rev. Cell Dev. Biol. 1997, 13, 203-229.

(9) Whippo, C. W.; Hangarter, R. P. Phototropism: Bending towards Enlightenment. Plant Cell 2006, 18, 1110-1119.

(10) Muday, G. K. Auxins and Tropisms. J. Plant Growth Regul. 2001, 20, 226-243.

(11) Enders, T. A.; Strader, L. C. Auxin activity: Past, present, and future. Am. J. Bot. 2015, 102, 180-196.

(12) Esmon, C. A.; Tinsley, A. G.; Ljung, K.; Sandberg, G.; Hearne, L. B.; Liscum, E. A gradient of auxin and auxin-dependent transcription precedes tropic growth responses. Proc. Natl. Acad. Sci. U. S. A. 2006, 103, 236-241.

(13) Dasog, M.; Carim, A. I.; Yalamanchili, S.; Atwater, H. A.; Lewis, N. S. Profiling Photoinduced Carrier Generation in Semiconductor Microwire Arrays via Photoelectrochemical Metal Deposition. Nano Lett. 2016, 16, 5015-5021.

(14) Lanyon, H. P. D. Optical and Electrical Properties of SeleniumTellurium Alloys. J. Appl. Phys. 1964, 35, 1516-1523. 


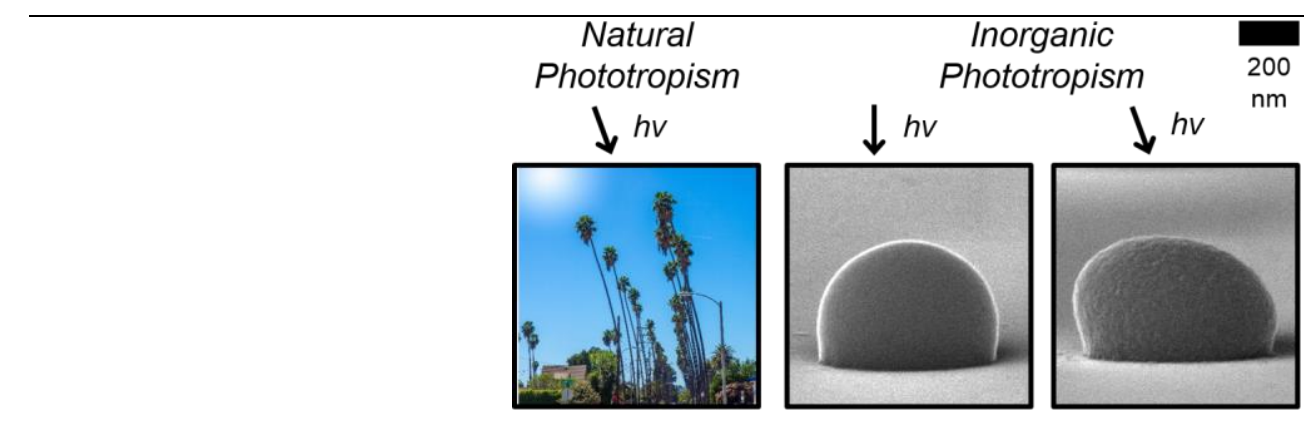

